\title{
Optimal Coordination of Vehicle-to-Grid Batteries and Renewable Generators in A Distribution System
}

\author{
Lu Wang*, Suleiman Sharkh, Andy Chipperfield \\ Faculty of Engineering and the Environment, University of Southampton, SO17 1BJ, UK
}

\begin{abstract}
The increasing penetration of electric vehicles (EVs) and renewable generators (RGs) in the power grid is an inevitable trend to combat air pollution and reduce the usage of fossil fuels. This will challenge distribution networks, which have constrained capacity. However, appropriate dispatch of electric vehicles via vehicle-to-grid (V2G) operation in coordination with the distributed renewable generators can provide support for the grid, reduce the reliance on traditional fossil-fuel generators and benefit EV users. This paper develops a novel agent-based coordinated dispatch strategy for EVs and distributed renewable generators, taking into account both grid's and EV users' concerns and priorities. This optimal dispatch problem is formulated as a distributed multi-objective constraint optimisation problem utilizing the Analytic Hierarchy Process and is solved using a dynamic-programming-based algorithm. The proposed strategy is tested on a modified UK Generic Distribution System (UKGDS). The electricity network model is simplified using a virtual sub-node concept to alleviate the computation burden of a node's agent. Simulation results demonstrate the feasibility and stability of this dispatch strategy.
\end{abstract}

Keywords: Vehicle-to-grid, distributed generators, optimal coordination, decentralized dispatch, dynamic programming.

\section{Nomenclature}

\footnotetext{
*Email Address: lw5g13@soton.ac.uk
} 


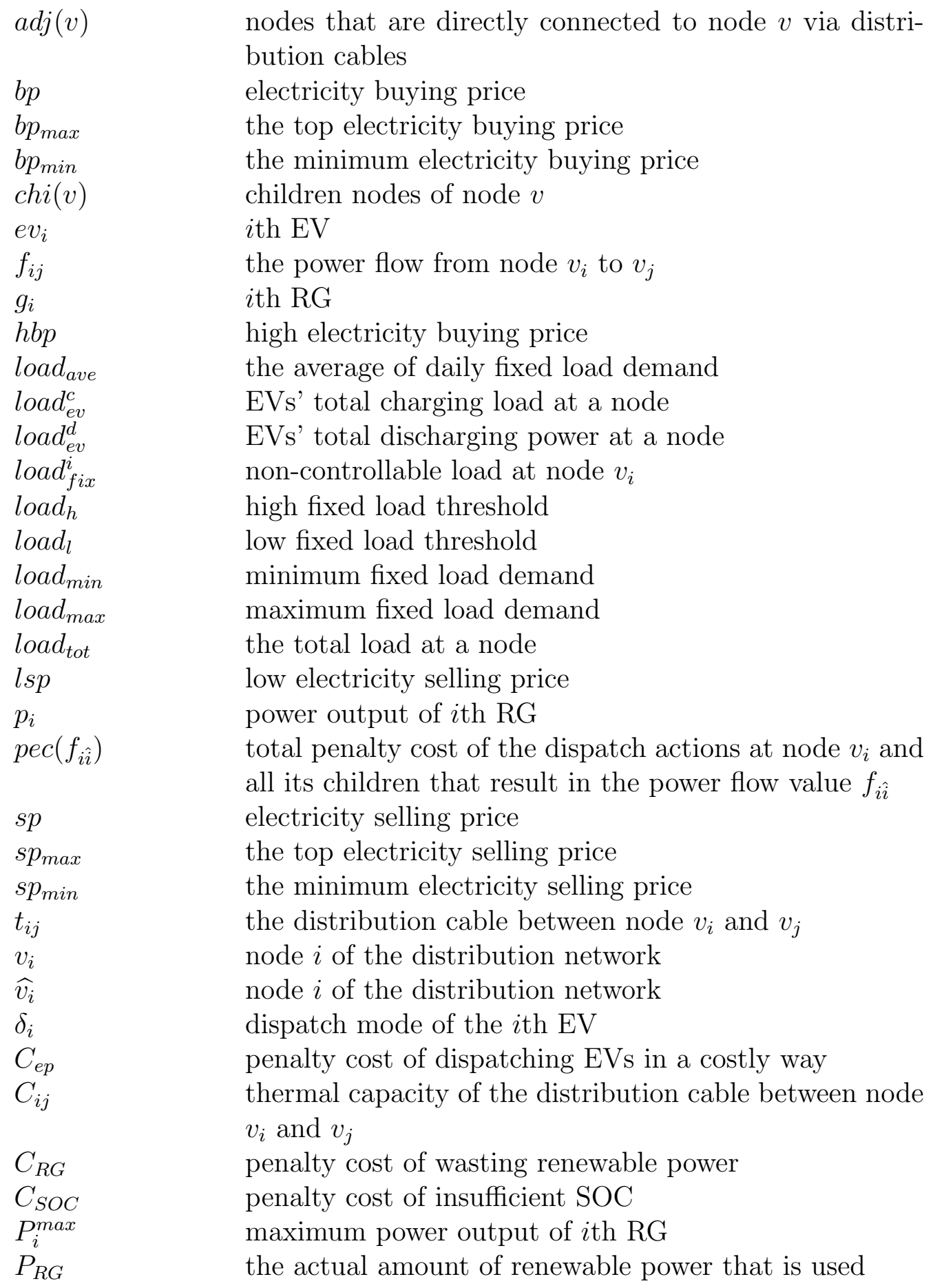




\begin{tabular}{|c|c|}
\hline$\widetilde{P_{R G}}$ & maximum available renewable power \\
\hline$P C_{a}$ & $\begin{array}{l}\text { the pairwise comparison matrix for the AHP model of } \\
\text { agents that have both EVs and RGs }\end{array}$ \\
\hline$P C_{b}$ & $\begin{array}{l}\text { rison matrix for the AHP model of } \\
\text { ly EVs }\end{array}$ \\
\hline $\mathrm{OC}$ & \\
\hline$f P$ & lessage \\
\hline & interval \\
\hline & me interval \\
\hline & e EV battery before \\
\hline & $\begin{array}{l}\text { ime before departure in multiples } \\
\text { ne interval }\end{array}$ \\
\hline Toparent & $\begin{array}{l}\text { an array of } P f P c \text { messages sent from node } v_{i} \text { 's agent to } \\
\text { its parent agent }\end{array}$ \\
\hline LinkToPfDstate & $\begin{array}{l}\text { Dispatch states at node } v_{i} \text { and all its children that result } \\
\text { in the corresponding } \operatorname{PfPc} \text { message formed at node } v_{i}\end{array}$ \\
\hline & \\
\hline
\end{tabular}

\section{Introduction}

Renewable generators (RGs) are increasingly replacing traditional fossil fuels to help meet the global requirement of air pollution alleviation and carbon emission reduction[1]. However, without effective management, those intermittent RGs could raise serious issues in the electricity network [2], such as imbalance between supply and demand, network overload and waste of the surplus renewable power.

As traffic results in about $26 \%$ of the worldwide $\mathrm{CO}_{2}$ emissions, electric vehicles (EVs) will increasingly penetrate into the power grid to replace fueldriven vehicles and realize clean transport $[1,3,4,5]$. The global EV market is forecast to grow from 1.7 million units in 2012 to 5.3 million units in 2020 [6]. At this rate, the number of EVs is set to increase considerably. This large-scale penetration of EVs will challenge the power system as an additional load due to the substantial need for battery charging, but it could help to increase the penetration of intermittent renewable energy sources by absorbing the extra electricity generated from these sources and then feeding it into the grid when needed $[7,8]$. By applying an optimal coordinated dispatch strategy of EVs with V2G operation and RGs, those issues that they might cause can be addressed. Furthermore, they may be even capable 
of providing operational support to the power grid and decreasing the total electric power production cost $[9,10]$.

Many papers have discussed strategies for dispatch of EV batteries when they are connected to the grid. An algorithm was proposed in [11] for optimal charging of EVs formulated as a stochastic dynamic programming problem taking into account the intrinsic uncertainty of their travel pattern. A charging optimization approach was proposed in [12] to maximize the total electric energy that all EVs absorb from the grid while avoiding violations of voltage limits and components' loading. Risk-aware day-ahead scheduling and realtime dispatch algorithms were developed for EV charging by Yang et al. [13] to minimize overall charging costs and the risk of EV load mismatch with the scheduled demand. A rule-based EV battery dispatch strategy consisting of three rule sets was demonstrated by Ma et al. [14], where the battery characteristics, SOC and electricity buying/selling prices were considered when determining the dispatch action (i.e. charge/discharge) and the rate of dispatch (i.e. charge/discharge current). The management of EV batteries has also been discussed in the literature $[15,16,17,18,19]$. However, as demonstrated in [20], in order to effectively reduce the $\mathrm{CO}_{2}$ emissions and reap desired environmental benefits, RGs should provide at least a part of EVs' charging energy.

A conceptual framework of wind-EV coordination is developed by $\mathrm{Li}$ et al. [1], which includes three-level hierarchy, to minimize the total grid operational cost including emission cost. However, this algorithm is used for day-ahead scheduling rather than real-time dispatch and requires centralized management. An optimal scheduling of EV charging was discussed by Zhang et al. [21] to minimize the mean waiting time of EVs at a renewablepower-aided charging station under cost constraint. However, vehicle-to-grid operation was not considered. Gao et al. [22] developed a hierarchical control structure for the dispatch of V2G batteries in the presence of RGs, in order to minimize operating cost. However, the proposed framework is applied to day-ahead scheduling of EV charging and discharging power instead of realtime operation. A control algorithm was proposed in [7] where wind power is used to minimize the generation cost; EVs are dispatched to smooth out fluctuations in wind power and improve the system's frequency regulation. However, the algorithm doesn't take into account the geographical location of the vehicle and associated local network constraints. The concept of virtual power plant (VPP) is utilized in [23], where VPPs consist of wind generators and EVs, to maximize the profits of selling electricity in the market. 
However, this methodology is mainly based on day-ahead and off-line optimization; it is not a real-time dispatch approach. Network constraints are not taken into account either. Evangelos et al. [24] proposed a distributed price-based algorithm so that EVs are coordinated with RGs and system's load levelling is achieved. However, network constraints are not considered in the model. It is a day-ahead scheduling approach instead of real-time dispatch, and the algorithm is not fully decentralized as in this model an agent requires the data from the whole network, e.g. total load demand, total DG power and SOC of EV batteries etc.

Most of the publications mentioned above, as well as [25, 26, 27, 28], focus on the centralized dispatch of a power network, which requires an awareness of the whole network. That could be problematic when faced with a large network with many renewable generators whose power outputs depend on unpredictable nature elements like wind and sunlight that could change at any time. Moreover, the number of constraints grow exponentially when the network becomes large [29], therefore at the end it will be nearly impossible to solve an optimal dispatch problem in a centralized way using available computer. Using a decentralized approach to solve the dispatch problem address the shortcomings of centralized dispatch, namely, the exponential growth of the computational and information gathering effort which render centralized dispatch to be unsuitable for real-time application. A decentralized approach shares the computational and information gathering effort amongst several agents, which solve the dispatch problem more efficiently. Such a system also has the advantage over a centralized approach in terms of expandability. It can be readily extended when the network expands or changes by including additional agents leaving the rest of the agents largely unchanged. Some researchers have developed partially decentralized dispatch approaches $[24,30,31,32]$. But they either neglected the capacity constraints or used a recursive strategy where the number of recursion increases exponentially with the increasing penetration of distributed generators. There is therefore a clear gap in the research with regards to the application of decentralized system to solving the coordinated dispatch of RGs and EV batteries, including $\mathrm{V} 2 \mathrm{G}$.

Therefore, a comprehensive coordinated dispatch strategy for both EVs and RGs is yet to be developed. Such a strategy needs to be decentralized and consider both EV users interests and grid operational requirements. Focusing on the different requirements of EV users and the grid will inevitably result in different dispatch patterns of EV batteries and RGs. Thus, every requirement 
needs to be weighted according to its relative importance when determining the dispatch mode of $\mathrm{EV}$ batteries and renewable generators.

In this work, a novel agent-based coordinated dispatch strategy for EVs and RGs is developed for real-time application, which aims at satisfying the concerns and requirements of both EV users and the grid, including 1. cost, as saving charging cost is a very common request of EV users, which are taken into account in many publications $[13,14,18,19]$; 2 . sufficient SOC for the next journey, which is important because mobility is the basic function of an EV, as considered in many researches $[1,11,13,19,21] ; 3$. improved utilization of renewable energy, because it is crucial for carbon emission reduction, as discussed earlier and in $[1,5,7,8] ; 4$. load levelling, which is one of the main grid operational support that EV batteries can provide and is also discussed in many papers $[9,15,19,24]$. In this strategy, each node in the network is represented by an software agent which is only aware of the elements that are locally connected to it and manages the dispatch of EVs and RGs connected to it, based on information received from the agents of other nodes that are directly linked to it, so that the stability of the network is ensured and all the objectives of dispatch are best achieved.

Being aware of a very large computational burden that could occur at a node's agent connecting with a great number of children nodes, a novel concept of a virtual sub-node is proposed to simplify the electricity network model in order to reduce this burden. Accordingly, the dispatch problem is formulated as a distributed multi-objective constraint optimization problem (DMOCOP) and then solved using a dynamic-programming-based algorithm to derive an optimal set of dispatch actions for EVs and RGs within a distribution network. The DMOCOP is developed from the distributed constraint optimization problem (DCOP) that was proposed in [33], using an Analytic Hierarchy Process (AHP) [34] to take several different objectives of dispatch, as discussed above, into account at the same time.

The proposed dispatch strategy is tested on a modified UKGDS, which is a radial distribution network, for its stability, feasibility and effectiveness at satisfying the requirements of both EV users and the grid. In practice, the aggregator or the distribution network operator (DNO) is supposed to be in charge of this optimal dispatch problem.

The rest of this paper is organized as follows. In Section 2, the proposed coordinated dispatch strategy of EVs and RGs is presented in detail. The results of simulations using MATLAB to verify its feasibility and efficacy, are presented and discussed in Section 3. Finally, the conclusions of the work 
are presented in Section 4.

\section{Coordinated Dispatch Strategy of Electric Vehicles and Renew- able generators}

The aim of the strategy is to realize optimal coordinated dispatch of EVs and RGs in the distribution network so that multiple objectives can be achieved such as saving charging cost to EV users while ensuring sufficient electricity remains in the batteries for the next journey, reducing waste of energy generated by RGs and supporting grid's operation like load levelling. It is assumed that the dispatch actions are conducted every 30 minutes.

The independent variables include the vehicles' driving patterns, which are initially randomly assigned as commonly done in many studies [17, 35]. The other independent variables including renewable power fluctuation, price of electricity and load pattern are determined based on historical data that can be found in [36, 37]. The dependent variables include cost, degree of utilization of RG, load levelling and SOC as discussed in Section 1.

\subsection{General Description of the Electricity Network and Agents}

Figure 1 shows a radial distribution network, which is derived from a UK Generic Distribution System (UKGDS)[38]. It includes renewable generators and EVs. The node $v_{0}$ is the slack bus, which is connected to the rest of power grid.

In more detail, the network contains a set of renewable generators, which are denoted by $\mathbf{G}=\left\{g_{1}, \ldots, g_{n}\right\}$ with each generator $g_{i}$ producing a certain amount of renewable power $p_{i} \in R G_{i}$ MW. $R G_{i}=\left\{0, \ldots, P_{i}^{\max }\right\}$, where $R G_{i}$ is discretized into $1 \mathrm{MW}$ steps to simplify the computational burden and $P_{i}^{\max } \in \mathbb{Z}^{+}$is the maximum power output of renewable generator $g_{i}$ rounded to the nearest whole $\mathrm{MW}$ at a particular moment in time; $P_{i}^{\max }$ will vary depending on the environmental conditions (e.g. cloud cover or wind speed variation). Let $\mathbf{p}=\left\{p_{1}, \ldots, p_{n}\right\}$ denote a set of power output variables for the renewable generators in $\mathbf{G}$.

Moreover, the network provides the capability of connecting a great number of EVs, each of which can be either charged or discharged. EV represents a set of EVs, where $\mathbf{E V}=\left\{e v_{1}, \ldots, e v_{m}\right\}$. Each EV has 7 choices of dispatch mode when it is parked and connected to the grid: charge $(+)$ or discharge $(-)$ at high (3), medium (2) or low-level (1) current, OR idle (0). Thus, each $\mathrm{EV} e v_{i}$ is dispatched in a certain mode $\delta_{i} \in D M_{i}=\{-3,-2,-1,0,1,2,3\}$, 
where the sign indicates the dispatch action (i.e. charge/discharge), and the absolute value represents the dispatch current level. Let $\boldsymbol{\delta}=\left\{\delta_{1}, \ldots, \delta_{m}\right\}$ denote a set of dispatch modes for EVs in $\mathbf{E V}$.

$V=\left\{v_{1}, \ldots, v_{k}\right\}$ denotes the set of nodes within the network. A node $v_{i}$ exchanges power with other nodes and contains a combination of fixed loads, EVs and RGs. $\operatorname{chi}\left(v_{i}\right)$ denotes a set of children nodes of $v_{i}$, while $\operatorname{adj}\left(v_{i}\right)$ represents a set of adjacent nodes of $v_{i}$, i.e., nodes that are directly connected to $v_{i}$ via distribution cables, including its children nodes and its parent node. load $_{\text {fix }}^{i}$ represents the fixed load at $v_{i}$, while $\mathbf{E V}\left(v_{i}\right)$ and $\mathbf{G}\left(v_{i}\right)$ denote the sets of EVs and RGs connected at $v_{i}$, respectively. Note that $\mathbf{E V}\left(v_{i}\right)=\varnothing$ and $\mathbf{G}\left(v_{i}\right)=\varnothing$ respectively mean that $v_{i}$ contains no EV and no RG.

The set of distribution cables in the network is denoted by $\mathbf{T}$ and $t_{i j}$ refers to the distribution cable between nodes $i$ and $j$. The power flow along the cable $t_{i j}$ is denoted by $f_{i j}$, and cannot exceed the thermal capacity of the cable, $C_{i j}$.

Furthermore, the network is controlled by agents in a decentralized way. Each node $v_{i}$ is represented by an agent, which is aware of the output power domains of the local RGs, $\mathbf{G}\left(v_{i}\right)$, and all possible dispatch modes of the connected EVs, $\mathbf{E V}\left(v_{i}\right)$, and has control over the dispatch of renewable power output and EVs. Each agent has a utility function, carries out a part of the computation required to achieve optimal and stable operation of the network (i.e. utility computation) and communicates the computation results with its adjacent agents that map to the adjacent nodes $\operatorname{adj}\left(v_{i}\right)$ of its designated node $v_{i}$. In this work, the utility function maps to the penalty cost at the designated node, which takes into account multiple objectives, using the Analytic Hierarchy Process (AHP), and measures how well these objectives are achieved. Based on the above definitions, this optimal coordinated dispatch problem can be formulated as a distributed multi-objective constraint optimisation problem (DMOCOP), which is developed from the distributed constraint optimisation problem (DCOP) proposed in [33], after simplifying the model using the virtual sub-node concept proposed in the following section to relieve the computational burden of a certain agent.

\subsection{Simplification of The Model Using Virtual Sub-Node Concept}

As will be discussed in the following section, an agent of a node performs computations based on the information received from its children and conducts the communication with them and the parent node. In a large network 
a node $v_{i}$ may have a great number of children nodes, which increases the computational and communication burden. In order to solve this issue, $v_{i}$ is considered to consist of several virtual sub-nodes, each of which is connecting some of $v_{i}$ 's children nodes and controlled by a sub-agent of the agent that maps to $v_{i}$. The sub-agents work simultaneously, with each sub-agent undertaking a part of the computation dealing with the information sent from the children of the corresponding virtual sub-node. A diagram of the distribution network with virtual sub-nodes is shown in Figure 2. In these figures, $v_{1}^{1}, v_{1}^{2}$ and $v_{1}^{3}$ are the three virtual sub-nodes of $v_{1}$. The load connected to $v_{1}, \operatorname{load}_{\text {fix }}^{1}$, is connected directly to the parent node. Furthermore, the distribution cable between $v_{1}$ and $v_{0}$ is divided into three virtual sub-cables, whose thermal capacities are defined based on $C_{01}$ and $\operatorname{load}_{\text {fix }}^{1}$. As $\operatorname{load}_{\text {fix }}^{1}$ is the fixed load at node $v_{1}$ consisting of active load pload ${ }_{\text {fix }}^{1}$ and reactive load load $_{\text {fix }}^{1}$. The total complex power that can be transmitted via $v_{1}$ to its children is limited in both active (real) and reactive (imaginary) parts, as follows:

$$
\begin{aligned}
C p_{01}^{n}= & \frac{\sum_{s \in \operatorname{chi}\left(v_{1}^{n}\right)} C_{s 1}}{\sum_{d \in \operatorname{chi}\left(v_{1}\right)} C_{d 1}} \times\left(\text { pload }_{\text {fix }}^{1} \times \frac{\left(C_{01}-\left|\operatorname{load}_{\text {fix }}^{1}\right|\right)}{\left|\operatorname{load}_{\text {fix }}^{1}\right|}\right) \\
C q_{01}^{n}= & \frac{\sum_{s \in \operatorname{chi}\left(v_{1}^{n}\right)} C_{s 1}}{\sum_{d \in \operatorname{chi}\left(v_{1}\right)} C_{d 1}} \times\left(\text { qload }_{\text {fix }}^{1} \times \frac{\left(C_{01}-\left|\operatorname{load}_{\text {fix }}^{1}\right|\right)}{\left|\operatorname{load}_{\text {fix }}^{1}\right|}\right)
\end{aligned}
$$

where $C p_{01}^{n}$ and $C q_{01}^{n}$ are the capacities of the $n$th virtual sub-cable in terms of active and reactive power flows, respectively. $\operatorname{ch} i\left(v_{1}^{n}\right)$ is a set of child nodes of the virtual sub-node $v_{1}^{n}$, e.g. $\operatorname{chi}\left(v_{1}^{1}\right)=\left[v_{2}, v_{3}\right]$ in Figure 2. Similarly, $\operatorname{chi}\left(v_{1}\right)$ is a set of $v_{1}$ 's children, i.e. $\left[v_{2}, v_{3}, v_{5}, v_{7}, v_{9}, v_{11}, v_{12}\right]$. These 2 equations will be easier to interpret using Figure 3. In Figure 3, the total capacity of the virtual sub-cables should be $x=C_{01}-\left|\operatorname{load}_{\text {fix }}^{1}\right|$. The absolute vale of $l o a d_{\text {fix }}^{1}$ is used to cater for both absorbing loads and generators. The apparent power capacity of a virtual sub-cable $C_{01}^{n}$ is determined by multiplying $x$ by the ratio of the total capacity of the cables connecting $v_{1}^{n}$ to its children nodes, which equals $\sum_{s \in c h i\left(v_{1}^{n}\right)} C_{s 1}$, to the total capacity of all the cables connecting the original $v_{1}$ node to its children nodes, i.e., $\sum_{d \in \operatorname{chi}\left(v_{1}\right)} C_{d 1}$. Active and reactive 
power capacities are calculated by multiplying the apparent power capacity of each virtual sub-cable by $\frac{\text { load }_{\text {fix }}^{1}}{\left|l o a d_{f i x}^{1}\right|}$ and $\frac{q l o a d_{\text {fix }}^{1}}{\mid l o a d_{f i x}^{1}}$, respectively.

The validity of the virtual sub-node approach has been verified via mathematical analysis and simulation (Details are given in the Appendix A), which demonstrate that it does not compromise the optimal dispatch results in this work.

\subsection{Formulation of Distributed Multi-Objective Constraint Optimisation Prob- lem (DMOCOP)}

The DMOCOP extends the DCOP procedure described in [33] to multiobjective optimization problems. It contains three main elements:

1. Variables: a set of $h$ variables $X=\left\{x_{1}, \ldots, x_{h}\right\}$. In this work, $x_{i}$ can be a renewable generator's power output or an EV's battery dispatch mode. Thus, in this work $X=\{\mathbf{p}, \boldsymbol{\delta}\}$.

2. Domains: a set of finite domains $D=\left\{d_{1}, \ldots, d_{h}\right\}$, which include all possible values of variables $X$. In this work, $d_{i}$ can be represented as follows:

$$
d_{i}= \begin{cases}R G_{i} & \begin{array}{l}
\text { when } x_{i} \text { is a } \\
\text { renewable } \\
\text { generator }
\end{array} \\
D M_{i} & \begin{array}{l}
\text { when } x_{i} \text { is an } \\
\text { EV battery }
\end{array}\end{cases}
$$

3. Utilities: a set of $k$ utilities $U=\left\{U_{1}, \ldots, U_{k}\right\}$, each of which corresponding to an agent. In this work, $U_{i}$ maps to the penalty cost at agent $i$, i.e., how unsatisfactory a combination of dispatch actions is in terms of the objectives. They are formed by using the AHP, as discussed in the following section.

The objectives include saving cost while leaving sufficient charge in an EV's battery, network load levelling and reduction of wasted renewable energy.

Objective 1 ( $R E)$ : Reduce wasted renewable energy.

Assume that the weather forecast is accurate enough, and it is predicted that a renewable generator's power output $\widetilde{P_{R G}}$ is available during the following time interval of 30 minutes. The penalty cost of wasting renewable power $C_{R G}$ is measured as follows: 


$$
C_{R G}=\left(\widetilde{P_{R G}}-P_{R G}\right) / \widetilde{P_{R G}}
$$

where $P_{R G}$ is the actual amount of power that is injected by a $R G$ into the network.

Objective 2 ( $B S$ ): Sufficient EV battery SOC.

Assuming that EVs' travel patterns are available (either directly entered by the user or estimated based on information in their diary or passed travel history), then the expected SOC at the end of the current time interval $S O C_{p}$ is estimated to be:

$$
S O C_{p}=\left\{\begin{array}{lc}
S O C & \text { if EV has no } \\
& \text { travel plan during } \\
& \text { the next } 2 \text { hours } \\
& \text { if EV has travel } \\
& \text { plan within } 2 \text { hours } \\
& \text { and current SOC } \\
& \text { is not enough for } \\
& \text { its next journey } \\
& \mathrm{t}=1 \text { time interval }) \\
& \text { if EV has travel } \\
& \text { plan within } 2 \text { hours } \\
& \text { and has enough SOC } \\
\text { for its next journey }
\end{array}\right.
$$

When the EV is expected to travel within the next 2 hours, a linear interpolation is used to determine the value of $S O C_{p}$ as illustrated in Figure 4. $T_{p}$ is the available preparation time before the next journey in multiples of dispatch action time interval, i.e. multiples of 30 minutes. $S O C_{p f}$ is the desired SOC of the EV battery before the next journey, which may be lower than the current SOC value, in which case the $\mathrm{EV}$ will be able to participate in V2G operations.

The aim is to keep the SOC at about 0.5. The penalty cost of insufficient SOC $C_{S O C}$ is measured as follows:

1. If the EV has no travel plan during the next 2 hours and the battery is more than half full at the end of this time interval OR if the $\mathrm{EV}$ will be used in 2 hours and battery's SOC is not less than $S O C_{p}$ at the end of the time interval, then:

$$
C_{S O C}=0
$$


2. If the EV has no travel plan during the next 2 hours and the battery is less than half full at the end of this time interval, then:

$$
C_{S O C}=\frac{0.5-S O C_{n}}{0.5-S_{\min }}
$$

where $S O C_{n}$ is the battery's SOC after a time interval of charging/discharging. In this work, the lower limit of SOC $S_{\min }$ is selected to be 0.4 to provide a good margin above the absolute minimum of 0.3 .

3. If the EV has travel plans within 2 hours and the battery's $S O C_{n}$ is lower than $S O C_{p}$ :

$$
C_{S O C}=\frac{S O C_{p}-S O C_{n}}{S O C_{p}-S_{\min }}
$$

Objective $3(C C)$ : Save charging cost to EV users.

The penalty cost is sure to be low when an EV is charged at a low electricity buying price while discharged at a high selling price. And the penalty cost $C_{e p}$ could vary depending on the how fast the EV is charged/discharged, i.e., the charging/discharging current.

1. Charge at high current (i.e. $30 \mathrm{~A}$ ):

$$
C_{e p}=\left\{\begin{array}{cc}
\frac{b p-b p_{\min }}{h b p-b p_{\min }} & b p<h b p \\
1 & b p \geqslant h b p
\end{array} .\right.
$$

2. Charge at mid-level current (i.e. $10 \mathrm{~A})$ :

$$
C_{e p}=\left\{\begin{array}{cl}
\frac{\left|b p-\left(b p_{\min }+h b p\right) / 2\right|}{\left(h b p-b p_{\min }\right) / 2} & b p<h b p \\
1 & b p \geqslant h b p
\end{array} .\right.
$$

3. Charge at low current (i.e. $2 \mathrm{~A})$ :

$$
C_{e p}=\left\{\begin{array}{cl}
\frac{h b p-b p}{h b p-b p_{\text {min }}} & b p<h b p \\
0 & b p \geqslant h b p
\end{array},\right.
$$

where $b p$ is the electricity buying price. $b p_{\max }$ and $b p_{\min }$ are respectively the top and bottom buying prices, while $h b p$ is the high buying price threshold defined as $h b p=0.9 *\left(b p_{\max }-b p_{\min }\right)+b p_{\min }$. Equations $(9)-$ (11) give progressively lower penalties as the charging current reduces. Similarly, the penalty cost for selling is defined as follows: 
4. Discharge at high current (i.e. $30 \mathrm{~A})$ :

$$
C_{e p}=\left\{\begin{array}{cc}
\frac{s p_{\max }-s p}{s p_{\max }-l s p} & s p>l s p \\
1 & s p \leqslant l s p
\end{array} .\right.
$$

5. Discharge at mid-level current (i.e. $10 \mathrm{~A}$ ):

$$
C_{e p}=\left\{\begin{array}{cl}
\frac{\left|s p-\left(s p_{\max }+l s p\right) / 2\right|}{\left(s p_{\max }-l s p\right) / 2} & s p>l s p \\
1 & s p \leqslant l s p
\end{array} .\right.
$$

6. Discharge at low current (i.e. $2 \mathrm{~A})$ :

$$
C_{e p}=\left\{\begin{array}{cc}
\frac{s p-l s p}{s p_{\max }-l s p} & s p>l s p \\
0 & s p \leqslant l s p
\end{array} .\right.
$$

7. No dispatch (i.e. $0 \mathrm{~A})$ :

$$
C_{e p}=\left\{\begin{array}{cc}
0 & s p \leqslant l s p \& b p \geqslant h b p \\
1 & \text { otherwise }
\end{array}\right.
$$

where $s p$ is the electricity selling price. $s p_{\max }$ and $s p_{\min }$ are respectively the top and bottom selling prices, while $l s p$ is the low selling price threshold defined as $l s p=0.1 *\left(s p_{\max }-s p_{\min }\right)+s p_{\min }$. These price data can be derived from historical data [36].

Objective 4 (LL): Load levelling in the distribution network.

The daily fixed (non-controllable) load demand is assumed to be available for both peak and off-peak periods. With the integration of EVs and RGs, the peak load could be pulled up further and might result in some spikes, or high power could be generated in the network and transferred to the rest of the grid through the slack bus during the off-peak period. Therefore, one of the objectives of coordinated dispatch of EVs and RGs is peak shaving and valley filling, i.e. load levelling. The penalty cost of failing to level the load $C_{l l}$ is evaluated as follows:

$$
C_{l l}=\left\{\begin{array}{cc}
0 & \text { load }_{l} \leqslant \text { load }_{\text {tot }} \leqslant \operatorname{load}_{h} \\
\frac{\text { load }_{\text {tot }}-\text { load }_{h}}{\text { load }_{\text {max }}-\text { load }_{h}} & \text { load }_{h}<\text { load }_{\text {tot }}<\operatorname{load}_{\text {max }} \\
\frac{\text { load }_{l}-\text { load }_{\text {tot }}}{\text { load }_{l}-\text { load }_{\text {min }}} & \text { load }_{\text {min }}<\text { load }_{\text {tot }}<\operatorname{load}_{l}, \\
\frac{\text { load }_{\text {min }}}{\text { load }_{\text {tot }}} & 0<\text { load }_{\text {tot }} \leqslant \operatorname{load}_{\text {min }} \\
\frac{\text { load }_{\text {tot }}}{\text { load }_{\max }} & \text { load }_{\text {tot }} \geqslant \operatorname{load}_{\text {max }}
\end{array}\right.
$$


where $l_{o a d_{\text {min }}}$ and $l_{\text {oad }}$ max are respectively the minimum and maximum fixed load demand during a day. $\operatorname{load}_{h}$ and $\operatorname{load}_{l}$ are the high and low fixed load thresholds, respectively, and they are determined by:

$$
\begin{aligned}
\operatorname{load}_{h} & =\left(\operatorname{load}_{\text {max }}+\operatorname{load}_{\text {ave }}\right) / 2, \\
\operatorname{load}_{l} & =\left(\operatorname{load}_{\text {min }}+\operatorname{load}_{\text {ave }}\right) / 2,
\end{aligned}
$$

where load $_{\text {ave }}$ is the average of daily fixed load demand. load $d_{t o t}$ is the total load at a node, which can be calculated by

$$
\operatorname{load}_{t o t}=\operatorname{load}_{f i x}+\operatorname{load}_{e v}^{c}-\operatorname{load}_{e v}^{d}-P_{R G},
$$

where $\operatorname{load}_{f i x}$ is the fixed load at a node, which is not controllable. $l o a d_{e v}^{c}$ and loadev are the EVs' charging load and the EVs' discharging power, respectively. $P_{R G}$ is the RG's output power.

\subsection{The Analytic Hierarchy Process}

In order to jointly consider these objectives and thus the corresponding penalty costs in the optimization process, they should be weighted depending on their relative importance in determining how EV batteries and renewable generators should be dispatched in coordination.

The Analytic Hierarchy Process (AHP) is a multi-criteria decision-making method developed by Saaty [34], which has been widely used in various areas including power systems [39, 40,41]. In this work, AHP is used to determine the priorities of different objectives of dispatch (i.e. the different requirements of users and grid). The main steps of the AHP methodology can be summarized as follows:

Step 1: A hierarchy model is established based on an analysis of the problem.

Step 2: Pairwise comparison matrices are formed at each level of the hierarchy model. Each element $A(i, j)$ in the pairwise comparison matrix $A$ depicts the expert's judgement of relative importance between the pair of $i t h$ and $j$ th factors using the ratio scale method [34, 41]. $A(i, j)$ is the reciprocal of $A(j, i)$.

Step 3: For each pairwise comparison matrix, the maximum eigenvalue and the corresponding eigenvector are calculated. The normalization of the calculated eigenvector gives the normalized principle eigenvector with elements equal to the priority scales (weightings) of the factors. 
Step 4: The consistency of judgements is checked. The Consistency Index $(C I)$ and Consistency Ratio $(C R)$ are measured for each pairwise comparison matrix. The $C I$ is defined as

$$
C I=\frac{\lambda_{\max }-n}{n-1}
$$

where $\lambda_{\max }$ and $n$ are the maximum eigenvalue and the dimension of the corresponding pairwise comparison matrix, respectively. The $C R$ is defined as

$$
C R=\frac{C I}{R I}
$$

where $R I$ is a set of given average random consistency indices which can be obtained using statistical calculations [40]. If $C R$ is no larger than $10 \%$, the inconsistency of judgements is acceptable. Otherwise, the judgements need to be revised.

Two different hierarchy models are respectively built up for the agents that only involve EV battery variables and those that have both EV battery and renewable generator variables, as shown in Figure 5.

The priorities of these agents' objectives are determined by forming a pairwise comparison matrix for each of the AHP hierarchy models shown in Figure 5; the scale numbers in the matrices are determined by the aggregator in practice based on experience and common sense. The matrices $P C_{a}$ (Figure 5(a)) and $P C_{b}$ (Figure 5(b)) are:

$$
\begin{aligned}
& R E \quad B S \quad C C \quad L L \\
& P C_{a}=\begin{array}{l}
R E \\
B S \\
C C \\
L L
\end{array}\left(\begin{array}{cccc}
1 & \frac{1}{2} & 2 & 2 \\
2 & 1 & 2 & 2 \\
\frac{1}{2} & \frac{1}{2} & 1 & 1 \\
\frac{1}{2} & \frac{1}{2} & 1 & 1
\end{array}\right) \\
& B S \quad C C \quad L L \\
& P C_{b}=\begin{array}{ll}
B S \\
& C L \\
L L
\end{array}\left(\begin{array}{ccc}
1 & 2 & 2 \\
\frac{1}{2} & 1 & 1 \\
\frac{1}{2} & 1 & 1
\end{array}\right) .
\end{aligned}
$$

By calculating the principle eigenvectors of $P C_{a}$ and $P C_{b}$, the priorities of $R E, B S, C C$ and $L L$ are found to be $27.81 \%, 39.52 \%, 16.34 \%$ and $16.34 \%$, 
respectively for AHP model (a), and the priorities of $B S, C C$ and $L L$ are respectively $50 \%, 25 \%$ and $25 \%$ for AHP model (b).

The utilities $U$ of agents that involve both EV battery and RG variables are then calculated as follows:

$$
\begin{aligned}
U= & 27.81 \% \times C_{R G}+39.52 \% \times C_{S O C} \\
& +16.34 \% \times C_{e p}+16.34 \% \times C_{L L},
\end{aligned}
$$

while the utilities $U$ of agents that only involve EV battery variables are calculated as follows:

$$
U=50 \% \times C_{S O C}+25 \% \times C_{e p}+25 \% \times C_{L L} .
$$

\subsection{Constraints}

For the stability of the electricity network and the better performance of EVs and RGs, several constraints are applied. The goal of agents is to find an assignment $X^{*}$ for the variables in $X$ (i.e. a combination of dispatch actions of EVs and RGs) that minimises the sum of penalty costs (i.e., the sum of utilities):

$$
\underset{X^{*}}{\arg \min } \sum_{i=0}^{k} U_{i},
$$

subject to the following constraints:

Constraint 1: The sum of power flow into a node $v_{i}$ should be equal to the sum of power flow out:

$$
\sum_{j \in a d j\left(v_{i}\right)} f_{i j}+\operatorname{load}_{f i x}+\operatorname{load}_{e v}^{c}-\operatorname{load}_{e v}^{d}-P_{R G}=0
$$

where $\operatorname{adj}\left(v_{i}\right)$ is the set of nodes that are connected to the node $v_{i} . f_{i j}$ is the power flow from node $i$ to $j$, and $f_{i j}=-f_{j i}$.

Constraint 2: The power flow along a distribution cable should not exceed its capacity:

$$
\left|f_{i j}\right| \leqslant C_{i j}
$$

where $C_{i j}$ is the thermal capacity of the distribution cable between nodes $v_{i}$ and $v_{j}$.

Constraint 3: The SOC of EV batteries should be within the range from 0 to 1 :

$$
0 \leqslant S O C \leqslant 1
$$


Constraint 4: When the EV is not going to be used within the next 2 hours and the battery is currently less than half full of electricity, the EV has to be charged:

$$
S O C_{n} \geqslant S O C, \quad \text { if } S O C<0.5
$$

Constraint 5: When the EV has a travel plan within the next 2 hours and the battery's SOC is currently less than desired at the end of the time interval, the EV has to be charged:

$$
S O C_{n} \geqslant S O C, \quad \text { if } S O C<S O C_{p}
$$

\subsection{Dynamic Programming Decentralized Optimal Dispatch (DPDOD)}

In DPDOD, the agent representing a particular node computes the utility function corresponding to that node according to (24) or (25). A variable can only be assigned to an agent subject to the rule that an agent controls the EV battery and RG variables locally at its designated node.

Phase 1 - Value Calculation:

The calculation starts from leaf nodes (i.e., nodes that have no children) and ends at the root node (i.e. the node that has no parent node). Only after it receives all the computed results from its children does a node start its own computation. After that it sends its computing results to its parent node.

For each agent $i$ (except agent 0 that controls $v_{0}$ ), the penalty cost is calculated for every possible combination of EV battery and RG dispatch actions, as well as the resulting power transfer along the distribution cable from the controlled node $v_{i}$ to its parent node $\widehat{v}_{i}$. Hence, a Power Flow and the associated Penalty Cost $(P f P c)$ message is formed as:

$$
\operatorname{PfPc}=<f_{i \hat{i}}, \operatorname{pec}\left(f_{i \hat{i}}\right)>,
$$

where $f_{i \hat{i}}$ is the power flow from $v_{i}$ to $\widehat{v}_{i} \cdot \operatorname{pec}\left(f_{i \hat{i}}\right)$ is the total penalty cost of the dispatch action combinations at $v_{i}$ and all its children that result in the power flow value $f_{i \hat{i}}$. Every $P f P c$ is checked and deleted if an alternative $P f P c$ exists with the same $f_{i \hat{i}}$ but smaller $\operatorname{pec}\left(f_{i \hat{i}}\right)$. Thus, the remaining $P f P c$ messages are those that record the minimum penalty cost that can be achieved for the specific $f_{i \hat{i}}$ 's, which are then formed into an array Toparent $_{i \rightarrow \hat{i}}$ defined as:

$$
\text { Toparent }_{i \rightarrow \hat{i}}=\left[P f P c_{1}, \ldots, P f P c_{m}\right] .
$$


Furthermore, each $P f P c$ in the Toparent ${ }_{i \rightarrow \hat{i}}$ maps to a LinkToPfDstate, which describes the dispatch actions of EV batteries and RGs at $v_{i}$ and the $P f P c$ messages of all its children that result in the total penalty cost described in the corresponding $P f P c$ message. Due to the different properties of nodes, there is a slight difference between the ways of construction of their $P f P c$ messages and LinkToPfDstate, which is explained below.

As leaf nodes have no child nodes, they only need to consider their own EV battery and RG dispatch actions when constructing their $P f P c$ messages. For each possible combination of dispatch actions at a leaf node $v_{i}$, a $P f P c$ message is constructed with power flow $f_{i \hat{i}}$ calculated as:

$$
f_{\hat{i}}=-\operatorname{load}_{t o t}=-\operatorname{load}_{f i x}-\operatorname{load}_{e v}^{c}+\operatorname{load}_{e v}^{d}+P_{R G} .
$$

The corresponding penalty cost is then calculated by (24), if $v_{i}$ has connections to both EVs and RGs, or (25), if $v_{i}$ only has EVs connected to it. As discussed earlier, some $P f P c$ messages are filtered out due to alternative $P f P c$ messages available with the same $f_{i \hat{i}}$ 's but lower penalty cost $\operatorname{pec}\left(f_{\hat{i} \hat{i}}\right.$ 's. Furthermore, each remaining $P f P c$ message maps to a LinkToPfDstate which records the corresponding EV battery and RG dispatch actions.

For a node $v_{j}$ that has at least one child node, all the Toparent arrays that it receives from its children $\operatorname{chi}\left(v_{j}\right)$ are considered along with its own EV and RG dispatch actions to compute its own Toparent Th $_{j \rightarrow \hat{j}}$ and construct the corresponding LinkToPfDstate. For each possible combination of the dispatch actions of the $\mathrm{EV}$ batteries and RGs at $v_{i}$ along with every possible combination of the $P f P c$ messages received from its children (with one from each child's Toparent array), the power flow $f_{j \hat{j}}$ is calculated as:

$$
\begin{aligned}
f_{j \hat{j}} & =-\operatorname{load}_{t o t}+\sum_{c \in \operatorname{chi}\left(v_{j}\right)} f_{c j} \\
& =-\operatorname{load}_{f i x}-\operatorname{load}_{e v}^{c}+\operatorname{load}_{e v}^{d}+P_{R G}+\sum_{c \in \operatorname{chi}\left(v_{j}\right)} f_{c j},
\end{aligned}
$$

where $\sum_{c \in c h i\left(v_{j}\right)} f_{c j}$ is the sum of power flows recorded in the chosen $P f P c$ messages from each of $v_{j}$ 's children. For each resultant power flow $f_{j \hat{j}}$, the minimum penalty cost that can realized is thus:

$$
\min _{f_{j \hat{j}}} \operatorname{pec}\left(f_{j \hat{j}}\right)
$$


where $\operatorname{pec}\left(f_{j \hat{j}}\right)$ is defined by:

$$
\operatorname{pec}\left(f_{j \hat{j}}\right)=U_{j}+\sum_{c \in \operatorname{chi}\left(v_{j}\right)} \operatorname{pec}\left(f_{c j}\right)
$$

where $\sum_{c \in \operatorname{chi}\left(v_{j}\right)} \operatorname{pec}\left(f_{c j}\right)$ is the sum of penalty costs calculated in the chosen P f Pc messages from each of $v_{j}$ 's children. $U_{j}$ is the penalty cost calculated at $v_{j}$ for a chosen combination of $\mathrm{EV}$ battery and RG dispatch actions, by using either (24) or (25) depending on whether EVs or RGs are connected to $v_{j}$. Each $P f P c$ message maps to a LinkToPfDstate, which also records the corresponding EV battery and RG dispatch actions at $v_{j}$ as well as the associated combination of $P f P c$ messages, one from each child.

Phase 2 - Value Propagation:

Once Phase 1 has been completed, and the root node has received the Toparent arrays from all of its children, its agent starts to examine every possible combination of the $P f P c$ messages, one from each Toparent array, in terms of whether the demand can be balanced with the supply and which combination minimizes the total penalty cost. A combination is considered to be feasible if the required power output from the root node to satisfy all the corresponding loads within the network is within its given feasible domain. Therefore, the feasible combination that minimizes the total penalty cost is selected as the optimum state of the network. Then, the power flow values from each of its $P f P c$ messages are sent to all of the root node's children, telling them which of their $P f P c$ messages minimize the total penalty cost. The child retrieves the correct $P f P c$ message that has the same power flow value as that received from the root node. Thus, its corresponding LinkToPfDstate specifies the optimal way to dispatch the $\mathrm{EV}$ batteries and RGs at this node, as well as the $\mathrm{PfPc}$ messages whose power flow values need to be sent to the corresponding children of this node. By iterating this procedure, the power flow values are propagated to the leaf nodes at the end. Thus, all nodes in the network know in which way their $\mathrm{EV}$ batteries and RGs should be dispatched to minimize the total penalty cost and best satisfy the objectives.

The operating procedure of DPDOD are concretely illustrated, taking a certain node's agent as an example, in Figure 6. 


\section{Simulations}

The proposed coordinated dispatch strategy of EV batteries and RGs was tested on the modified generic radial distribution network shown in Figure 1. In this distribution network, four renewable generators are located at nodes $v_{3}, v_{6}, v_{11}$ and $v_{12}$, respectively. Moreover, $33000 \mathrm{EVs}$ are assumed to exist in the network with each node, except $v_{0}$ and $v_{1}$, capable of connecting 3000 EVs to the grid at the same time. All the network data, including thermal capacity of distribution cables, as shown in Table 1. The fixed load at each node, in Table 2, are derived from UKGDS model [38]. The parameters of EV batteries are obtained from [14]. The simulation is implemented on a laptop with a Dual Core 2GHz CPU and 8GB RAM using MATLAB.

The total load demand and system selling/buying prices of electricity during a day were taken from [36]. The total load demand is scaled down so that the peak demand during a day is $350 \mathrm{MW}$, which approximately represents the daily load demand in a typical UK regional distribution network, as shown in Figure 7. Due to scarcity of information on the payments by/to EV users when the EVs are charged/discharged, the system selling/buying prices are adjusted based on domestic tariffs to represent EV users' selling/buying prices of electricity [14]. The adjusted system selling/buying price is derived by adding the difference between the average tariff value and the average system selling/buying price to the corresponding system selling/buying price, as shown in Figure 8:

$$
A S P(t)=S P(t)+\left(\frac{\sum_{t=1}^{T} \operatorname{tariff}(t)}{T}-\frac{\sum_{t=1}^{T} S P(t)}{T}\right)
$$

where a day is divided into $T$ time intervals. $A S P(t)$ and $S P(t)$ are the adjusted system selling/buying price and system selling/buying price at time interval $t$, respectively, while tarif $f(t)$ represents the domestic tariff value at time interval $t$. Therefore, the ASSP (i.e. adjusted system selling price) and ASBP (i.e. adjusted system buying price) in Figure 8 are respectively used as the EV charging and discharging prices, namely the $b p$ and $s p$ in Objective 3, respectively.

Furthermore, the renewable generators erected in this network are assumed to be wind turbines, and the daily weather forecast is assumed to be 
accurate enough. The historical wind power data recorded in [37], as shown in Figure 9, is used in the simulations.

As for the EVs' travel patterns, they are randomly generated based on the probability of parked cars during a weekday as shown in Figure 10. When the $\mathrm{EV}$ is on the road, the battery electric energy is assumed to be consumed at the nominal discharging current (20A) as assumed in [14]. Furthermore, the initial SOC of EVs are randomly assigned with a normal probability distribution $(\mu=0.6, \sigma=0.1)$. The time step is set to be 30 minutes. The dispatch actions are determined by the dispatch strategy at the beginning of every time interval and lasts for the entire time interval of 30 minutes, as mentioned earlier.

The simulation was run several times, each time starting with a different randomly assigned SOC, to determine the net costs to EV users (i.e. charging costs-discharging payments) during a day, as shown in Table 3. In the table, the average daily charging cost of $33000 \mathrm{EVs}$ within the network is shown to be $£ 11835$ in total (by simply summing up the average daily cost at every node), thus the average daily cost of each $\mathrm{EV}$ is calculated to be only $£ 0.36$, compared to a cost of $£ 1.53$ per day per vehicle when EV is charged in an uncontrolled way (based on simulations assuming that EVs are charged when their SOCs are less than 0.8 and renewable energy is utilized as much as possible without overloading the cables). However, the EVs parked at certain nodes tend to cost more than the EVs at the other nodes and the amount of costs/payments within the same node also varies, depending on the driving patterns of EVs and their initial SOCs. Furthermore, the simulations confirmed that the dispatch strategy ensures that EVs can complete their daily journeys without running out of electricity on the road and always ensures that enough energy (over $31 \%$ of battery's available capacity) remains in their batteries, as demonstrated in Table 4. Figure 11 presents an EV's SOC variation during a day under the proposed dispatch strategy, which includes 2 driving activities from 8:30 to 9:30 and from 14:00 to 14:30.

As for the renewable generators, one of the aims of this dispatch strategy is to utilize as much of their energy as possible, i.e., wind energy in this work. The simulation result shown in Figure 12 (Case 1, where the objectives' priorities are given in (24)) demonstrates consistency with this aim, i.e., the daily usage rate of wind power within the network is calculated to be $100 \%$, with no wind power wasted. Compared to Case 1, if the relative priority of objective $R E$ is lowered (i.e., Case 2 in Figure 12: priorities of $R E, B S, C C$, $L L$ are $14.04 \%, 33 \%, 19.96 \%$ and $33 \%$ for example), $1.4 \%$ of wind power 
cannot be directly absorbed and utilized by the network if no extra energy storage device is located at the RG. This occurs because when the $R E$ 's priority is relatively low, the strategy will choose to save charging cost to EV users and achieve valley filling at the expense of abandoning some of the wind power and using less energy to charge EVs, as expected.

Moreover, the changes of network load demand due to the integration of EVs and RGs are demonstrated in Figure 13, where positive values refer to load decrease (peak shaving) while negative values imply load increase (valley filling). It is shown in Figure 13, that a significant load levelling is fulfilled by the coordinated dispatch strategy, given the relatively small EV storage, in comparison with uncontrolled dispatch of EVs and RGs. From this figure, it is clear to see that peak shaving and valley filling has been realized by the coordinated integration of EVs and RGs for the distribution network's daily load demand, and the coordinated dispatch results in a better performance than the uncontrolled one.

To verify the stability of the proposed coordinated dispatch strategy, the simulation described above continues running to check EVs and RGs' performance for 7 days. The 7-day load curves of the network with the integration of EVs and RGs are shown in Figure 14, from which it is obvious that load levelling is well achieved and the load curves for the 7 days approximately overlay each other, meaning that the dispatch system is very stable. The EV SOCs at the end of a day are checked as well to see whether they remain in a similar normal distribution curve from day to day with similar mean and standard deviation values, as shown in Figure 15 and Table 5. Moreover, when EVs start with different assignments of initial SOCs, they will stabilise at a very similar normal distributions of their SOCs at the end of every day (i.e. approximately $\mu=0.57, \sigma=0.22$ ).

\section{Conclusion}

The feasibility and stability of the proposed agent-based control strategy was verified by the simulation results. The performance of EVs and RGs under the proposed coordinated dispatch strategy is stable. Furthermore, the normal distribution of EVs' daily final SOCs is very similar from day to day, even when different initial SOCs are assigned, which also verifies the stability of the proposed dispatch strategy. On average it costs $£ 0.36$ per vehicle per day, and all journeys are completed leaving the battery with at least 31\% SOC. But individual vehicles connected at different nodes may 
have higher or lower costs due to their locations, travel patterns and initial SOC. The cost/payment to an EV owner may vary from day to day due to different initial SOC of the day. A $100 \%$ of the wind power generated is absorbed by the network. EVs and RGs cooperate well to achieve load levelling of the network's load demand.

The saving per day of just over $£ 1$ per vehicle is small and it may be argued that this is not a sufficient incentive for drivers to participate in such a scheme given the impact it may have on their vehicles' battery life and availability. However, drivers will be well advised to consider the other important and indirect benefits. If they don't participate in such a coordinated charging scheme they may find that they are not able to charge their batteries or even have electricity in their homes due to the network reaching its peak capacity as a result of uncoordinated charging. Participating in the scheme will ensure that they will continue to enjoy uninterrupted supply of electricity. They will also benefit from the increase of the utilization of renewable energy and the corresponding reduction in fossil fuel emissions - they will basically have a cleaner environment, fresh air and fewer worries about global warming and other environmental concerns. If they don't participate and the distribution network operator (DNO) is forced to upgrade the system, the EV owners will end up paying more for their electricity to cover the cost of the upgrade.

In the current market condition, there are obstacles to the implementation and realization of the full potential of the proposed optimal coordination methodology of EVs and RGs. For example, the financial benefits to EV users may not be a sufficient incentive for participating in the V2G scheme. EV users and RG owners may not wish to cooperate with DNOs. Profit driven DNOs may prefer conventional solutions of network reinforcement over the risky $\mathrm{V} 2 \mathrm{G}$. But the market will continue to evolve driven by economic, political and social factors, which may improve the prospects for $\mathrm{V} 2 \mathrm{G}$ and coordinated RG and V2G dispatch. The proposed methodology can be readily adjusted and evolved to accommodate new market conditions.

\section{Appendix A. Proof of Virtual Sub-Node Concept}

In order to verify that using the virtual sub-node concept to simplify the model will not compromise the optimal dispatch results in this work, it needs to be proved that the following two optimization problems are equivalent (i.e., result in the same optimal solutions): 
Problem 1:

$$
\begin{array}{ll} 
& \min \sum_{c \in c h i\left(v_{1}\right)} \operatorname{pec}\left(f_{c 1}\right) \\
\text { s.t. } & \sum_{c \in c h i\left(v_{1}\right)} f_{c 1} \leqslant C_{01}
\end{array}
$$

Problem 2:

$$
\begin{aligned}
& \min \sum_{c \in \operatorname{chi}\left(v_{1}^{n}\right)} \operatorname{pec}\left(f_{c 1}\right) \\
\text { s.t. } \quad & \sum_{c \in \operatorname{chi}\left(v_{1}^{n}\right)} f_{c 1} \leqslant C_{01}^{n}, \quad n=1,2,3 . \\
& \sum_{n=1}^{3} C_{01}^{n}+\operatorname{load} d_{f i x}^{1}=C_{01} \\
C_{01}^{1}: C_{01}^{2}: C_{01}^{3}= & \sum_{d \in \operatorname{chi}\left(v_{1}^{1}\right)} C_{d 1}: \sum_{d \in \operatorname{chi}\left(v_{1}^{2}\right)} C_{d 1}: \sum_{d \in \operatorname{chi}\left(v_{1}^{3}\right)} C_{d 1} .
\end{aligned}
$$

First of all, because the three virtual sub-nodes are independent, it is clear that:

$$
\begin{array}{ll} 
& \min \sum_{c \in c h i\left(v_{1}\right)} \operatorname{pec}\left(f_{c 1}\right)=\sum_{n=1}^{3}\left(\min \sum_{c \in \operatorname{chi}\left(v_{1}^{n}\right)} \operatorname{pec}\left(f_{c 1}\right)\right) \\
\text { s.t. } & \sum_{c \in \operatorname{chi}\left(v_{1}\right)} f_{c 1} \leqslant C_{01},
\end{array}
$$

which can be easily proved by contradiction. Then, the next step is to prove that problem 2 shares the same optimal solution with the following problem:

$$
\begin{array}{ll} 
& \min \sum_{c \in c h i\left(v_{1}^{n}\right)} \operatorname{pec}\left(f_{c 1}\right) \\
\text { s.t. } & \sum_{c \in c h i\left(v_{1}\right)} f_{c 1} \leqslant C_{01},
\end{array}
$$

which is an equivalent substitute of problem 1 thus denoted as problem 1'.

As the proposed simplification process (i.e., problem 2) is adding more constraints on the optimization problem compared to problems 1 and 1', 
the optimum solved from problems 1 or 1 ' might be cut out. Therefore, a direct way is to prove that the global optimum stays within the constraints of problem 2. By running the simulation to confirm that the optimal solution of problem 2 is the same as that of the unconstrained optimization problem:

$$
\min \sum_{c \in \operatorname{chi}\left(v_{1}^{n}\right)} \operatorname{pec}\left(f_{c 1}\right),
$$

the global optimum is verified to meet the constraints of problem 2 , that is, the optimal solutions of problem 1, 1' and 2 are all the same and equal to the global optimum. In other words, as long as the capacities of the virtual sub-cables are set such that they are in proportion to the total capacities of the downstream cables, as defined in (1) and (2), this simplification process realizes the equivalence in terms of deriving the same optimal dispatch solution while reducing the computation burden of the agent.

\section{References}

[1] Z. Li, Q. Guo, H. Sun, Y. Wang, S. Xin, Emission-concerned windev coordination on the transmission grid side with network constraints: Concept and case study, IEEE Transactions on Smart Grid 4 (3) (2013) 1692-1704.

[2] M. Zipf, D. Most, Impacts of volatile and uncertain renewable energy sources on the German electricity system, in: 10th International Conference on the European Energy Market (EEM), Sweden, 2013, pp. 1-8.

[3] A. Foley, B. Tyther, P. Calnan, B. O. Gallachoir, Impacts of electric vehicle charging under electricity market operations, Applied Energy 101 (0) (2013) $93-102$.

[4] F. Millo, L. Rolando, R. Fuso, F. Mallamo, Real CO2 emissions benefits and end user's operating costs of a plug-in hybrid electric vehicle, Applied Energy 114 (0) (2014) 563 - 571.

[5] A. Verma, R. Raj, M. Kumar, S. Ghandehariun, A. Kumar, Assessment of renewable energy technologies for charging electric vehicles in Canada, Energy 86 (2015) $548-559$. 
[6] Global EV market forecast, [Online], Available: http://www . greencarcongress. com/2012/12/anderman-20121218.html (Dec 2012).

[7] C.-T. Li, C. Ahn, H. Peng, J. Sun, Synergistic control of plug-in vehicle charging and wind power scheduling, IEEE Transactions on Power Systems 28 (2) (2013) 1113-1121.

[8] D. Dallinger, S. Gerda, M. Wietschel, Integration of intermittent renewable power supply using grid-connected vehicles - a 2030 case study for california and germany, Applied Energy 104 (0) (2013) 666 - 682.

[9] H. Fathabadi, Utilization of electric vehicles and renewable energy sources used as distributed generators for improving characteristics of electric power distribution systems, Energy 90, Part 1 (2015) 1100 1110 .

[10] M. Carrion, R. Zarate-Minano, Operation of renewable-dominated power systems with a significant penetration of plug-in electric vehicles, Energy 90, Part 1 (2015) 827 - 835.

[11] E. B. Iversen, J. M. Morales, H. Madsen, Optimal charging of an electric vehicle using a markov decision process, Applied Energy 123 (0) (2014) $1-12$.

[12] P. Richardson, D. Flynn, A. Keane, Optimal charging of electric vehicles in low-voltage distribution systems, IEEE Transactions on Power Systems 27 (1) (2012) 268-279.

[13] L. Yang, J. Zhang, H. Poor, Risk-aware day-ahead scheduling and realtime dispatch for electric vehicle charging, IEEE Transactions on Smart Grid 5 (2) (2014) 693-702.

[14] Y. Ma, T. Houghton, A. Cruden, D. Infield, Modeling the benefits of vehicle-to-grid technology to a power system, IEEE Transactions on Power Systems 27 (2) (2012) 1012-1020.

[15] M. Singh, P. Kumar, I. Kar, Implementation of vehicle to grid infrastructure using fuzzy logic controller, IEEE Transactions on Smart Grid 3 (1) (2012) 565-577. 
[16] J. Lopes, F. Soares, P. Almeida, Integration of electric vehicles in the electric power system, Proceedings of the IEEE 99 (1) (2011) 168-183.

[17] M. Yazdani-Damavandi, M. Moghaddam, M.-R. Haghifam, M. Shafiekhah, J. Catalao, Modeling operational behavior of plug-in electric vehicles' parking lot in multienergy systems, IEEE Transactions on Smart Grid PP (99) (2015) 1-1.

[18] O. Erdinc, N. Paterakis, T. Mendes, A. Bakirtzis, J. Catalao, Smart household operation considering bi-directional ev and ess utilization by real-time pricing-based dr, IEEE Transactions on Smart Grid 6 (3) (2015) 1281-1291.

[19] L. Hua, J. Wang, C. Zhou, Adaptive electric vehicle charging coordination on distribution network, IEEE Transactions on Smart Grid 5 (6) (2014) 2666-2675.

[20] N. Masuch, J. Keiser, M. Lutzenberger, S. Albayrak, Wind power-aware vehicle-to-grid algorithms for sustainable ev energy management systems, in: 2012 IEEE International Electric Vehicle Conference (IEVC), 2012, pp. 1-7.

[21] T. Zhang, W. Chen, Z. Han, Z. Cao, Charging scheduling of electric vehicles with local renewable energy under uncertain electric vehicle arrival and grid power price, IEEE Transactions on Vehicular Technology 63 (6) (2014) 2600-2612.

[22] S. Gao, K. Chau, C. Liu, D. Wu, C. Chan, Integrated energy management of plug-in electric vehicles in power grid with renewables, IEEE Transactions on Vehicular Technology 63 (7) (2014) 3019-3027.

[23] M. Vasirani, R. Kota, R. L. G. Cavalcante, S. Ossowski, N. R. Jennings, An agent-based approach to virtual power plants of wind power generators and electric vehicles, IEEE Transactions on Smart Grid 4 (3) (2013) 1314-1322.

[24] E. L. Karfopoulos, N. D. Hatziargyriou, Distributed coordination of electric vehicles providing v2g services, IEEE Transactions on Power Systems 31 (1) (2016) 329-338. 
[25] C. Wang, Y. Liu, X. Li, L. Guo, L. Qiao, H. Lu, Energy management system for stand-alone diesel-wind-biomass microgrid with energy storage system, Energy 97 (2016) 90 - 104.

[26] V. Mohan, J. G. Singh, W. Ongsakul, An efficient two stage stochastic optimal energy and reserve management in a microgrid, Applied Energy $160(2015) 28-38$.

[27] C. Chen, F. Wang, B. Zhou, K. W. Chan, Y. Cao, Y. Tan, An interval optimization based day-ahead scheduling scheme for renewable energy management in smart distribution systems, Energy Conversion and Management 106 (2015) $584-596$.

[28] M. Peik-herfeh, H. Seifi, M. K. Sheikh-El-Eslami, Two-stage approach for optimal dispatch of distributed energy resources in distribution networks considering virtual power plant concept, International Transactions on Electrical Energy Systems 24 (1) (2014) 43-63.

[29] M. Granada, M. Rider, J. R. Mantovani, M. Shahidehpour, Multi-areas optimal reactive power flow, in: 2008 IEEE/PES Transmission and Distribution Conference and Exposition: Latin America, Colombia, 2008, pp. 1-6.

[30] Y. Zhang, N. Gatsis, G. Giannakis, Robust energy management for microgrids with high-penetration renewables, IEEE Transactions on Sustainable Energy 4 (4) (2013) 944-953.

[31] C. xia Dou, W. qian Wang, D.-W. Hao, X. bin Li, Mas-based solution to energy management strategy of distributed generation system, International Journal of Electrical Power \& Energy Systems 69 (2015) 354 366.

[32] F. Ren, M. Zhang, D. Sutanto, A multi-agent solution to distribution system management by considering distributed generators, IEEE Transactions on Power Systems 28 (2) (2013) 1442-1451.

[33] S. Miller, S. D. Ramchurn, A. Rogers, Optimal decentralised dispatch of embedded generation in the smart grid, in: Proceedings of 11th International Conference on Autonomous Agents and Multi-Agent Systems (AAMAS), Spain, 2012, pp. 281-288. 
[34] T. L. Saaty, The Analytic Hierarchy Process, McGraw Hill, New York, 1980.

[35] M. Shafie-khah, J. P. S. Catalao, A stochastic multi-layer agent-based model to study electricity market participants behavior, IEEE Transactions on Power Systems 30 (2) (2015) 867-881.

[36] UK national grid, [Online], Available: http://www.bmreports.com/ bwx_reporting.htm (Jan 2014).

[37] UK national grid status, [Online], Available: http://www.gridwatch. templar.co.uk/index.php (Jan 2014).

[38] UK generic distribution network, [Online], Available: http://www. sedg.ac.uk/ukgds.htm (Oct 2013).

[39] D.-M. Kim, J.-O. Kim, Design of emergency demand response program using analytic hierarchy process, IEEE Transactions on Smart Grid 3 (2) (2012) 635-644.

[40] J. Zhu, M. Irving, Combined active and reactive dispatch with multiple objectives using an analytic hierarchical process, IEE ProceedingsGeneration, Transmission and Distribution 143 (4) (1996) 344-352.

[41] J. Zhu, J. A. Momoh, Optimal VAr pricing and VAr placement using analytic hierarchical process, Electric Power Systems Research 48 (1) (1998) $11-17$.

\section{Figures and Tables}




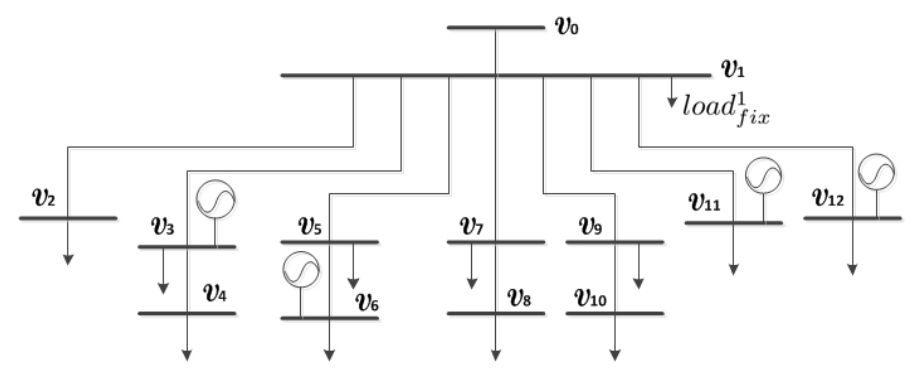

Figure 1: Diagram of a modified generic radial distribution network

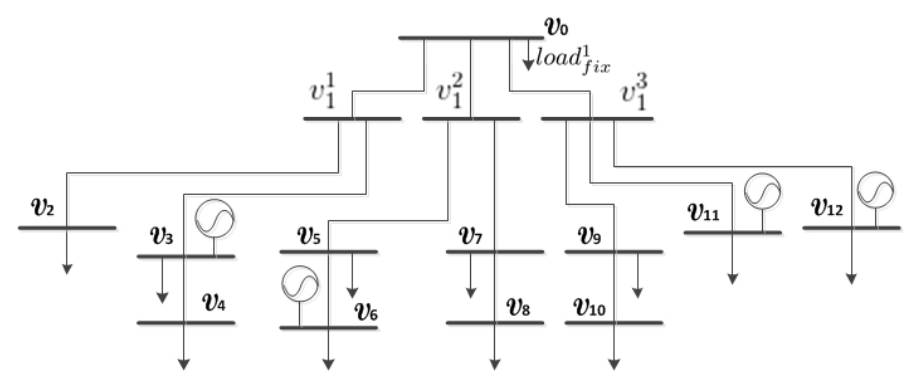

Figure 2: The diagram of the radial distribution network with virtual sub-nodes

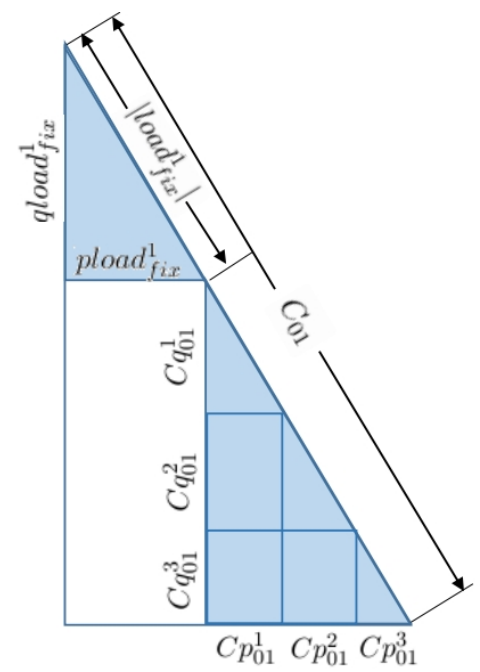

Figure 3: An illustration of how the virtual sub-cable capabilities are derived 


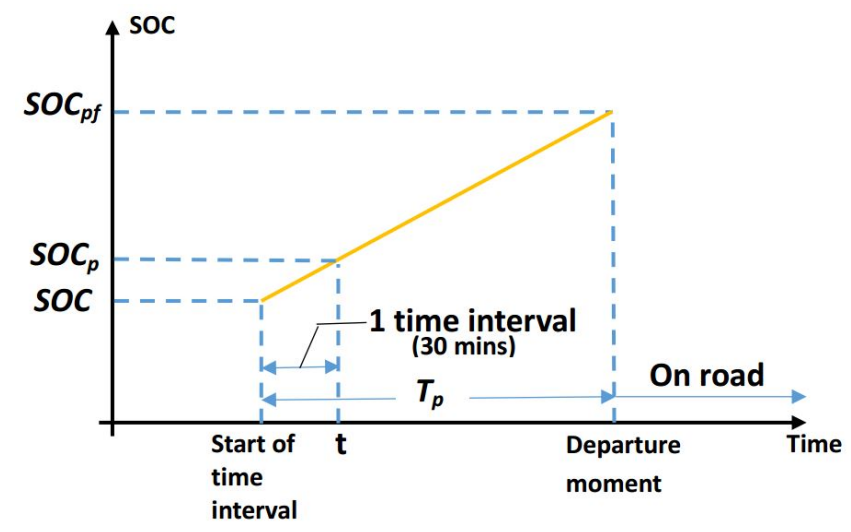

Figure 4: Illustration of the calculation of $S O C_{p}$ when EV has travel plan within 2 hours and current SOC is not adequate

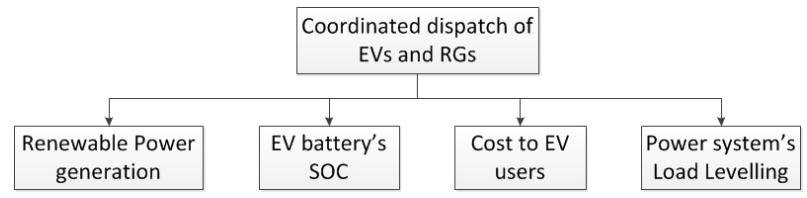

(a) Agents that have both EV battery and renewable generator variables

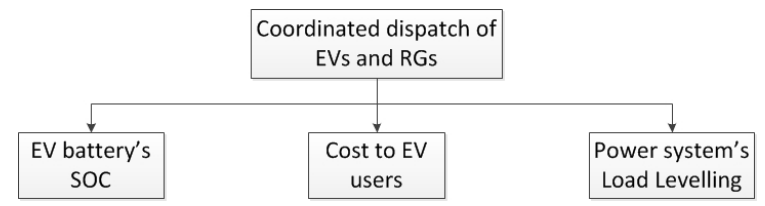

(b) Agents that involve EV battery variables only

Figure 5: AHP Hierarchy Models of the Utilities of Two Types of Agents for the Coordinated Dispatch of EVs and RGs 


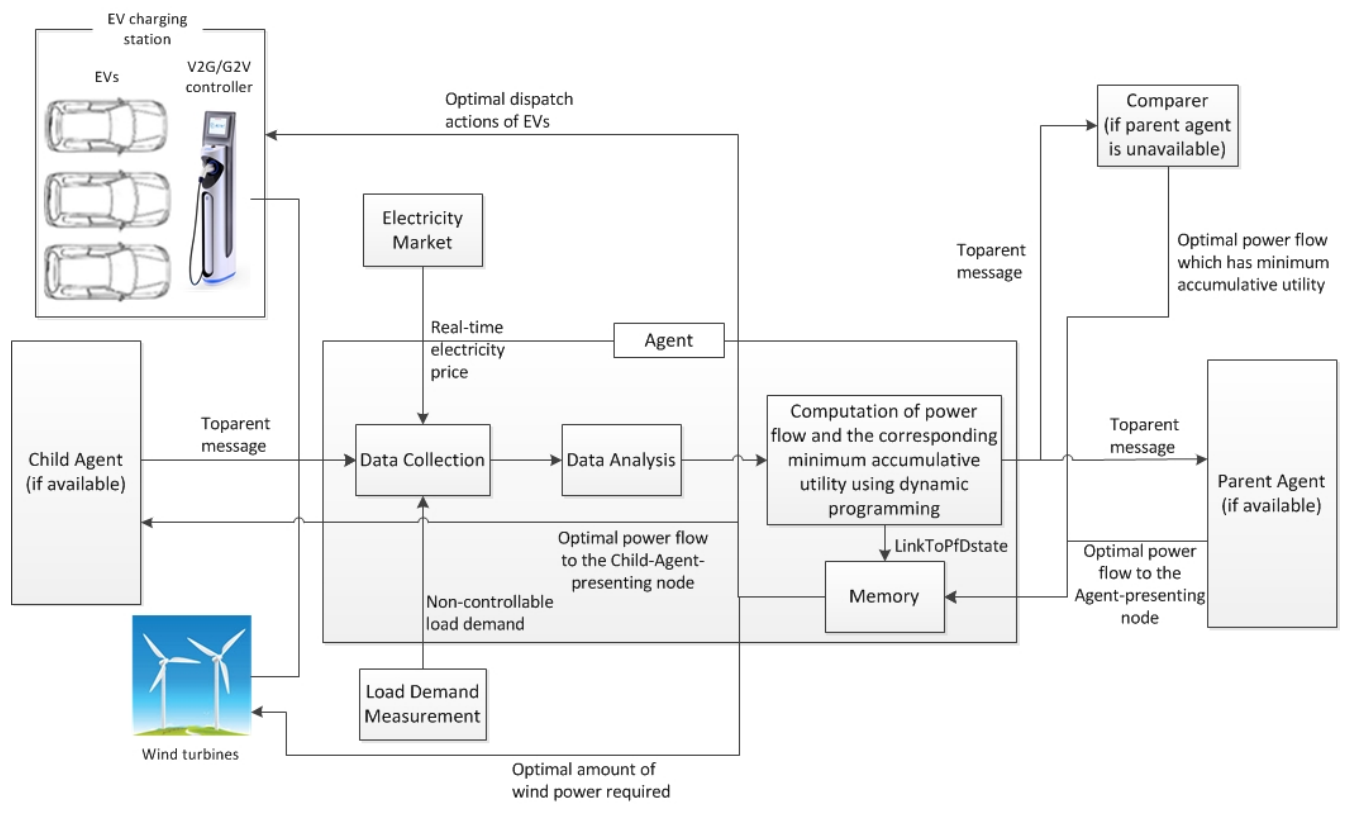

Figure 6: The Operation of DPDOD at A Certain Node's Agent

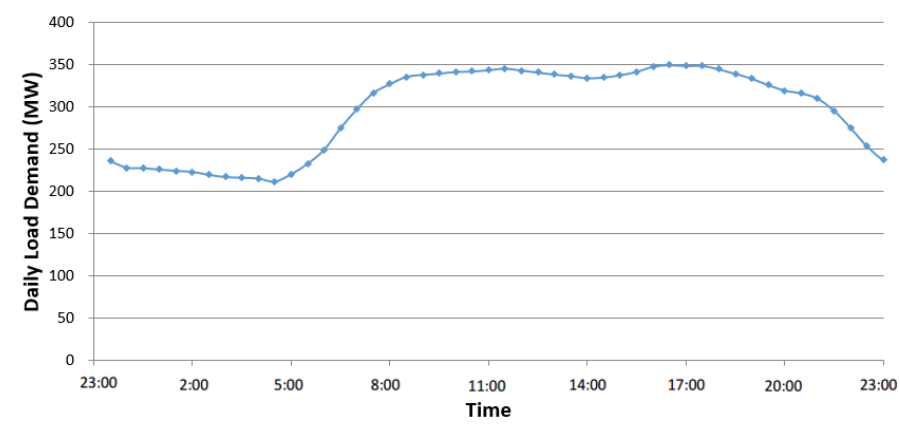

Figure 7: Total Fixed Load Demand During A Day 


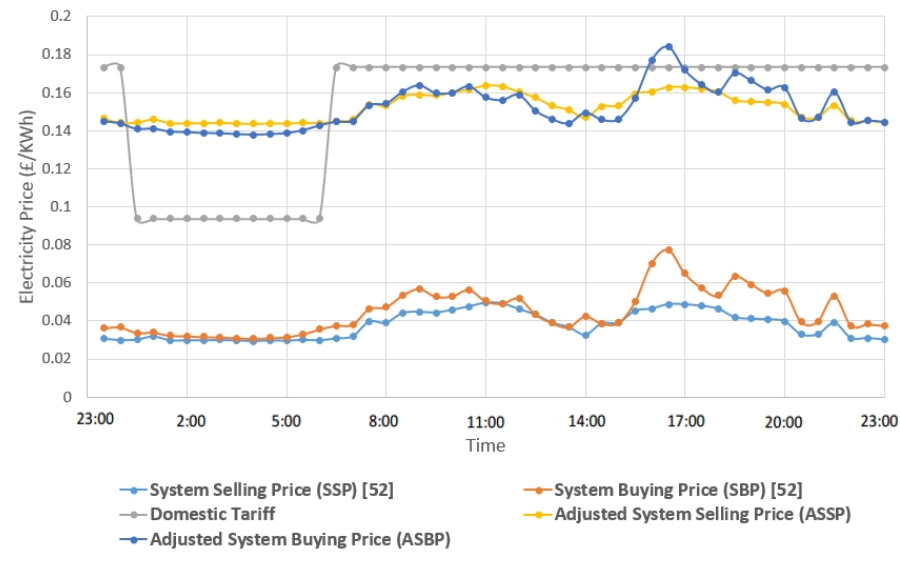

Figure 8: Daily Electricity Price

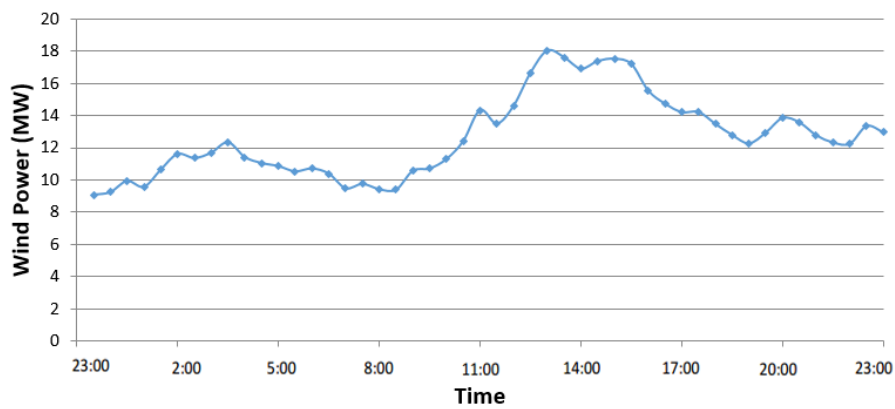

Figure 9: Daily wind power generated in the network

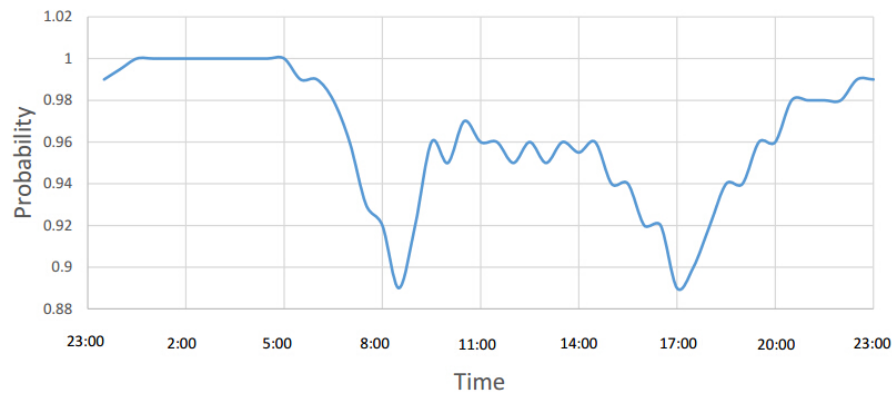

Figure 10: Probability of cars that are parked during a weekday [14] 


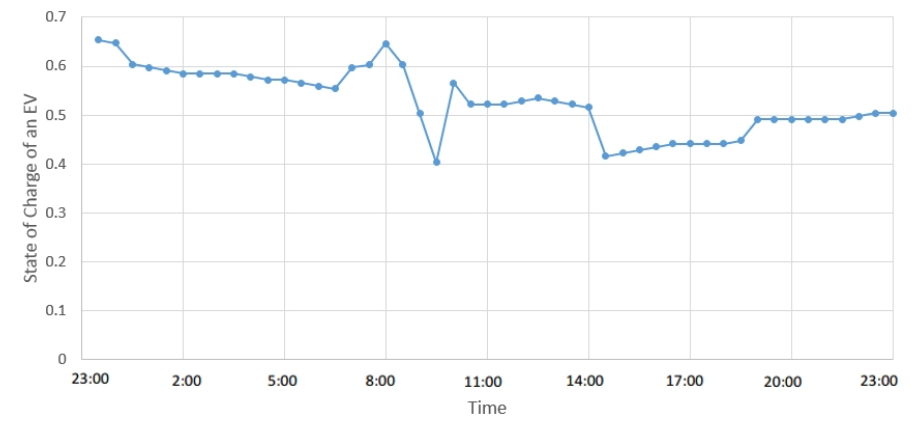

Figure 11: The variation of SOC of an EV at node 2

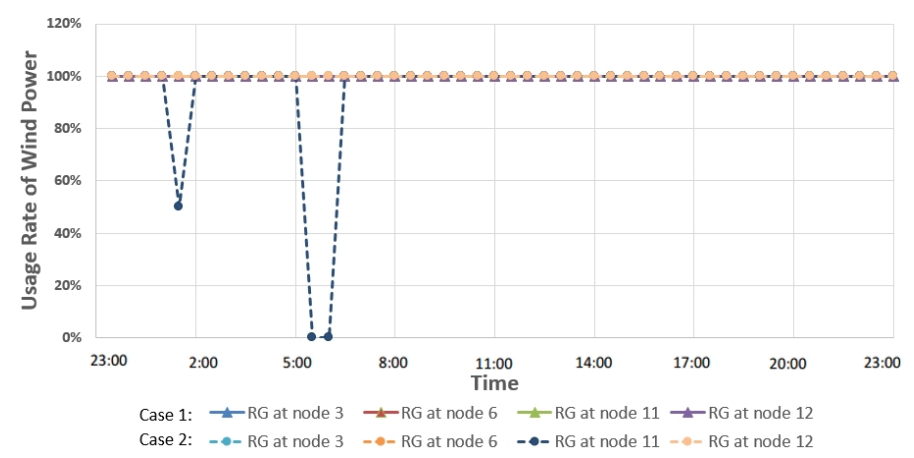

Figure 12: The usage rate of wind power at each renewable generator (Case 1: Relatively high priority is given to the objective $R E$, i.e. the case where priority settings are as demonstrated in (22) and (23). Case 2: Relatively low priority is given to the objective $R E$.)

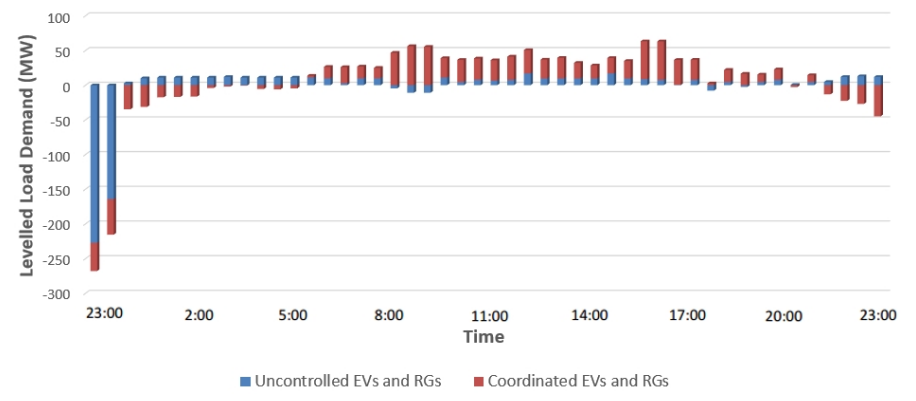

Figure 13: The change of total network's daily load demand that is caused by the integration of EVs and RGs in 2 different ways (i.e. uncontrolled and coordinated ways, respectively) 


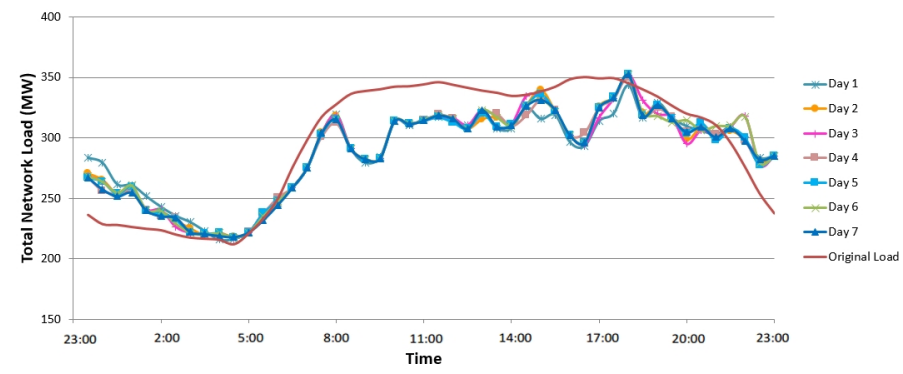

Figure 14: The load demand of the network within a week

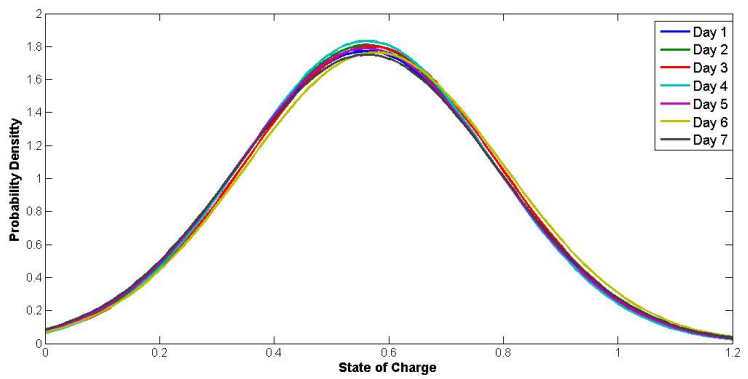

Figure 15: EVs' SOC distribution curves at the end of every day with their initial SOC randomly assigned with a normal probability distribution $(\mu=0.6, \sigma=0.1)$

Table 1: Thermal Capacity of Distribution Cables

\begin{tabular}{|c|c|c|}
\hline \multicolumn{2}{|c|}{ Distribution Cable } & $\begin{array}{c}\text { Thermal Capacity } \\
\text { (MVA) }\end{array}$ \\
\cline { 1 - 2 } From Node & To Node & \\
\hline$v_{0}$ & $v_{1}$ & 500 \\
\hline$v_{1}$ & $v_{2}$ & 52 \\
\hline$v_{1}$ & $v_{3}$ & 154 \\
\hline$v_{1}$ & $v_{5}$ & 102 \\
\hline$v_{1}$ & $v_{7}$ & 76 \\
\hline$v_{1}$ & $v_{9}$ & 76 \\
\hline$v_{1}$ & $v_{11}$ & 54 \\
\hline$v_{1}$ & $v_{12}$ & 154 \\
\hline$v_{3}$ & $v_{4}$ & 81 \\
\hline$v_{5}$ & $v_{6}$ & 110 \\
\hline$v_{7}$ & $v_{8}$ & 46 \\
\hline$v_{9}$ & $v_{10}$ & 51 \\
\hline
\end{tabular}


Table 2: Fixed Load at Each Node of the Distribution Network

\begin{tabular}{|c|c|c|}
\hline Node & $\begin{array}{c}\text { Fixed Active Load } \\
\text { (MW) }\end{array}$ & $\begin{array}{c}\text { Fixed Reactive Load } \\
\text { (MVAR) }\end{array}$ \\
\hline$v_{1}$ & 38.48 & 11.28 \\
\hline$v_{2}$ & 22.98 & 9.11 \\
\hline$v_{3}$ & 56.05 & 3.63 \\
\hline$v_{4}$ & 36.63 & 9.77 \\
\hline$v_{5}$ & 25.32 & 5.55 \\
\hline$v_{6}$ & 55.54 & 6.19 \\
\hline$v_{7}$ & 17.70 & 0 \\
\hline$v_{8}$ & 15.73 & 2.28 \\
\hline$v_{9}$ & 13.14 & 0 \\
\hline$v_{10}$ & 20.93 & 3.70 \\
\hline$v_{11}$ & 99.05 & 21.09 \\
\hline$v_{12}$ & 87.07 & 16.58 \\
\hline
\end{tabular}

Table 3: Daily costs of EVs calculated from simulations starting with different initial SOC

\begin{tabular}{|c|c|c|c|c|c|c|c|c|c|c|c|}
\hline \multirow{2}{*}{$\begin{array}{c}\text { Simulation } \\
\text { Run No. }\end{array}$} & \multicolumn{11}{|c|}{$\begin{array}{l}\text { Net Costs to EV users } \\
\qquad(£)\end{array}$} \\
\hline & $v_{2}$ & $v_{3}$ & $v_{4}$ & $v_{5}$ & $v_{6}$ & $v_{7}$ & $v_{8}$ & $v_{9}$ & $v_{10}$ & $v_{11}$ & $v_{12}$ \\
\hline 1 & 1830 & 1302 & -412 & -580 & -209 & 648 & -845 & 772 & 241 & 2442 & 935 \\
\hline 2 & 3596 & 2023 & -511 & 745 & 930 & -660 & 2133 & 286 & 156 & 2580 & 2171 \\
\hline 3 & 2725 & 357 & -148 & 1157 & 543 & -392 & 1447 & 1506 & 1095 & 2525 & 3057 \\
\hline 4 & 3131 & 1444 & -883 & -237 & 1605 & 348 & 1339 & -1073 & 581 & 3080 & 2663 \\
\hline 5 & 3063 & 890 & 152 & 1505 & 32 & 1682 & 1727 & 1090 & 1407 & 520 & 20 \\
\hline 6 & 3212 & 783 & 185 & 1286 & -328 & 1682 & 2100 & 1115 & 1377 & 1392 & 381 \\
\hline 7 & 2760 & 934 & -997 & 1364 & 225 & 1715 & 1866 & 1157 & 1373 & 266 & 524 \\
\hline 8 & 2673 & 1057 & -232 & 1286 & -209 & 1716 & 1825 & 1115 & 1374 & 845 & 350 \\
\hline 9 & 3092 & 769 & 218 & 1287 & -195 & 1683 & 2040 & 1115 & 1407 & 1976 & 934 \\
\hline 10 & 3114 & 888 & -866 & 1287 & -75 & 1683 & 1833 & 1149 & 1407 & 232 & -329 \\
\hline Average & 2920 & 1044 & -349 & 910 & 232 & 1010 & 1547 & 823 & 1042 & 1586 & 1071 \\
\hline
\end{tabular}

Table 4: minimum and maximum SOCs of EVs during a day

\begin{tabular}{|c|c|c|c|c|c|}
\hline Node & $\begin{array}{c}\text { Minimum } \\
\text { SOC }\end{array}$ & $\begin{array}{c}\text { Maximum } \\
\text { SOC }\end{array}$ & Node & $\begin{array}{c}\text { Minimum } \\
\text { SOC }\end{array}$ & $\begin{array}{c}\text { Maximum } \\
\text { SOC }\end{array}$ \\
\hline$v_{2}$ & 0.37 & 1.00 & $v_{3}$ & 0.36 & 1.00 \\
\hline$v_{4}$ & 0.40 & 1.00 & $v_{5}$ & 0.41 & 1.00 \\
\hline$v_{6}$ & 0.43 & 1.00 & $v_{7}$ & 0.33 & 0.99 \\
\hline$v_{8}$ & 0.33 & 1.00 & $v_{9}$ & 0.31 & 1.00 \\
\hline$v_{10}$ & 0.33 & 0.99 & $v_{11}$ & 0.44 & 1.00 \\
\hline$v_{12}$ & 0.42 & 0.99 & & & \\
\hline
\end{tabular}


Table 5: Key factors of EVs' SOC distribution at the end of every day tested with different mean values of the random assignments of the initial SOC

\begin{tabular}{|c|c|c|c|c|c|c|}
\hline \multirow{2}{*}{ Day } & \multicolumn{2}{|c|}{$\boldsymbol{\mu = 0 . 5}$} & \multicolumn{2}{c|}{$\boldsymbol{\mu = 0 . 6}$} & \multicolumn{2}{c|}{$\boldsymbol{\mu = 0 . 7}$} \\
\cline { 2 - 7 } & Mean & $\begin{array}{c}\text { Standard } \\
\text { deviation }\end{array}$ & Mean & $\begin{array}{c}\text { Standard } \\
\text { deviation }\end{array}$ & Mean & $\begin{array}{l}\text { Standard } \\
\text { deviation }\end{array}$ \\
\hline 1 & 0.57 & 0.22 & 0.56 & 0.23 & 0.56 & 0.22 \\
\hline 2 & 0.57 & 0.22 & 0.56 & 0.22 & 0.57 & 0.23 \\
\hline 3 & 0.57 & 0.22 & 0.57 & 0.22 & 0.56 & 0.22 \\
\hline 4 & 0.57 & 0.22 & 0.56 & 0.22 & 0.57 & 0.23 \\
\hline 5 & 0.56 & 0.22 & 0.56 & 0.22 & 0.57 & 0.22 \\
\hline 6 & 0.57 & 0.22 & 0.58 & 0.23 & 0.56 & 0.22 \\
\hline 7 & 0.57 & 0.22 & 0.56 & 0.23 & 0.56 & 0.22 \\
\hline
\end{tabular}

